TRANSACTIONS OF THE

AMERICAN MATHEMATICAL SOCIETY

Volume 356, Number 7 , Pages 2851-2870

S 0002-9947(03)03371-3

Article electronically published on July 24, 2003

\title{
METRICAL DIOPHANTINE APPROXIMATION FOR CONTINUED FRACTION LIKE MAPS OF THE INTERVAL
}

\author{
ANDREW HAAS AND DAVID MOLNAR
}

\begin{abstract}
We study the metrical properties of a class of continued fractionlike mappings of the unit interval, each of which is defined as the fractional part of a Möbius transformation taking the endpoints of the interval to zero and infinity.
\end{abstract}

\section{INTRODUCTION}

Working with an ergodic transformation $T$ of the interval that is given as a piecewise Möbius transformation, one comes to expect an associated continued fraction theory. The continued fraction expansion of a number $x$ is a concrete realization of the symbolic representation of the number as a sequence of locations within intervals of monotonicity of the iterates $T^{n}$. The piecewise Möbius nature of the map allows for a representation of the endpoints of the intervals of monotonicity nesting about $x$ as particular "fractions", $\frac{p_{n}}{q_{n}}$, called the convergents of $x$.

To offer a few familiar examples, the classical continued fraction theory may be seen as arising from a study of the Gauss map,

$$
G(x)=\left\langle\frac{1}{x}\right\rangle=\frac{1}{x}-\left[\frac{1}{x}\right],
$$

where $[a]$ denotes the integral part of $a$ and consequently $G(x)$ is the fractional part of $\frac{1}{x}$ 3, 9]. There are two other well-known continued fraction theories, one arising from the Renyi or backward continued fraction map $R(x)=\left\langle\frac{1}{1-x}\right\rangle$ [1, 17] and the other from the nearest integer map $H(x)=\frac{1}{x}-\left[\frac{1}{x}-\frac{1}{2}\right][16]$. $G$ and $R$ are defined on the unit interval, while $R$ is a self-map of $\left[-\frac{1}{2}, \frac{1}{2}\right]$. All are piecewise Möbius transformations and have actual rational fractions as convergents.

Several related families of mappings have been studied, with attention to their dynamical properties as well as to the resulting continued fraction theories [6, 10. 15 22. This fits into a broader series of connections which have been observed between continued fractions, diophantine approximation, ergodic theory and hyperbolic geometry, perhaps originating with Artin and more recently seen popping up all over 1, 2, 7, 8, 10. Most commonly the dynamical system is introduced as a tool for studying the continued fraction theory. Although there has been much progress, only in a few cases, for example see [20, 21], is it possible to achieve the incredibly refined results acheived in the classical "metrical" theory of continued fractions [3, 5, 13, 18].

Received by the editors July 8, 2002 and, in revised form, April 8, 2003.

2000 Mathematics Subject Classification. Primary 11J70, 11J83, 37E05.

Key words and phrases. Continued fractions, interval maps, diophantine approximation. 
We shall focus on the endomorphisms of the unit interval of the form $T(x)=$ $\langle A(x)\rangle$, where $A$ is a Möbius transformation taking one of the endpoints $\{0,1\}$ to 0 and the other to $\infty$. Not all such maps are ergodic or even recurrent. Among those that are, there is a realization of the natural automorphic extension of $T$ [9] acting on a subset of $\mathbb{R}^{2}$ for which the density $\frac{1}{(x-y)^{2}}$ defines an invariant measure. Except for two maps for which the invariant measure is infinite, the others are all Bernoulli with respect to the normalized measure [11]. Earlier work by Rudolfer and Wilkinson looked at the dynamic properties of a subclass of these mappings and showed them to be weak Bernoulli 22. Observe that both the Gauss and Rényi maps are among the ones being considered so far, although both are usually defined by a Möbius transformation taking one of the endpoints to 1 instead of 0 .

Our goal is to develop the basic continued fraction theory for the transformations with finite invariant measure and then to use the natural extension, in the manner pioneered by Bosma, Jager and Wiedijk [5] in the classical cases, to elucidate some fairly refined properties of the approximating convergents and consequently of the associated interval structure for the mappings. An important tool for our work, which distills a technique from [5], is Theorem 3. This result allows us to conclude convergence for ergodic sums in two variables almost everywhere in one of the variables.

For each of the mappings we shall derive what is classically known as the Khintchine constant, which gives the limiting geometric mean of a generic digit expansion. We shall also derive the Khintchine-Lévy constants. These describe the generic rate of growth of the denominators of convergents and can be used to determine something like the generic rate of shrinkage for nesting intervals of mononticity. Similar techniques allow the computation, for almost all $x$, of the values

$$
\lim _{n \rightarrow \infty} \frac{1}{n} \sum_{i=1}^{n} \frac{\left|x-\frac{p_{i}}{q_{i}}\right|}{\left|x-\frac{p_{i-1}}{q_{i-1}}\right|} \text { and } \lim _{n \rightarrow \infty} \frac{1}{n} \log \left|x-\frac{p_{n}}{q_{n}}\right|
$$

for all of the mappings. Several of these values relate simply and directly to the entropy of the mappings. The Euler dilogarithm makes a few notable appearances. Along the way we deduce a variety of diophantine, metrical and other properties of the continued fractions.

While the classical theory of the Gauss map is contained as a special case of our approach, the Rényi map is one of those with infinite invariant measure and therefore its convergents are not distributed in a way that can be understood using our methods.

\section{2. $u$-CONTINUED FRACTIONS AND DYNAMICS}

2.1. Matrices and maps: Basic definitions. A $2 \times 2$ matrix

$$
C=\left(\begin{array}{ll}
a & b \\
c & d
\end{array}\right)
$$

with real entries and determinant $a d-b c= \pm 1$ acts on the Riemann sphere $\mathbb{C} \cup\{\infty\}$ as a Möbius transformation by

$$
C(z)=\frac{a z+b}{c z+d}, C(\infty)=\frac{a}{c} \text { and } C\left(-\frac{d}{c}\right)=\infty .
$$

Such matrices preserve the extended real line $\mathbb{R} \cup\{\infty\}$ and either preserve or interchange the lower and upper half planes, depending on whether the determinant 
is positive or negative. Matrix multiplication goes over, in this setting, to the composition of the corresponding Möbius transformations [2].

We shall be considering the family of Möbius transformations parameterized by $\Psi \subset \mathbb{R} \times\{0,1\}$, where $\Psi$ is defined as the union of

$$
\Psi_{0}=((-\infty,-1) \cup(0, \infty)) \times\{0\} \text { and } \Psi_{1}=((-\infty, 0) \cup(1, \infty)) \times\{1\} .
$$

Define the matrices

$$
A_{(k, 0)}=\left(\begin{array}{cc}
\frac{k}{\sqrt{|k|}} & \frac{-k}{\sqrt{|k|}} \\
\frac{-1}{\sqrt{|k|}} & 0
\end{array}\right) \text { and } A_{(k, 1)}=\left(\begin{array}{cc}
\frac{k}{\sqrt{|k|}} & 0 \\
\frac{-1}{\sqrt{|k|}} & \frac{1}{\sqrt{|k|}}
\end{array}\right) .
$$

Written as Möbius transformations, these have the form

$$
A_{(k, 0)}(z)=\frac{k(1-z)}{z} \text { and } A_{(k, 1)}(z)=\frac{k z}{1-z} .
$$

The second coordinate of the parameterization indicates the endpoint of the interval $[0,1]$ that is mapped to $\infty$ by the transformation. The remaining endpoint always maps to zero. We use the notation $u=(k, m) \in \Psi$.

For any real number $t,[t]$ shall denote the greatest integer less than or equal to $t$, otherwise known as the integer part of $t$. For technical reasons, define the integer part of $\infty$ to be zero. The fractional part of $t$, written $\langle t\rangle$, is $t-[t]$. Also define $\langle\infty\rangle=0$. Let $I=[0,1]$, the closed unit interval. Then for $u \in \Psi$ the interval transformation $T_{u}: I \rightarrow I$ is the piecewise Möbius transformation

$$
T_{u}(x)=\left\langle A_{u}(x)\right\rangle .
$$

$T_{u}$ will be called Gauss-like when $u \in \Psi_{0}$ and Renyi-like when $u \in \Psi_{1} . T_{(1,0)}$ and $T_{(1,1)}$ are respectively the classical Gauss and Renyi transformations, also known as the continued fraction and backward continued fraction maps [1, 10]. These transformations provide a dynamical system approach for studying properties of the regular and backwards continued fractions. Another important distinction will often be made depending on whether the parameter $u$ satisfies either $(-1)^{m} k>$ 0 or $(-1)^{m} k<0$. As we shall see, this latter classification is more significant when considering the dynamical or "metrical" behavior of the transformations.

2.2. Intervals of monotonicity and finite continued fractions. Given $u \in \Psi$, the set of integers

$$
V_{u}=\left\{l \in \mathbb{Z} \mid\left[A_{u}(x)\right]=l \text { for some } x \in(0,1)\right\}
$$

are the $u$-digits. When $k>0, V_{u}$ is the set of non-negative integers and when $k<0$, it is the set of negative integers. $V_{u}$ should be thought of as the set of integers $a$ so that $A_{u}(x)-a \in(0,1)$ for some $x \in(0,1)$.

For each sequence of $n$ integers $a_{1}, \ldots, a_{n} \in V_{u}$, the open interval (cylinder set) at level $n$ is the image of the interval $(0,1)$ under a composition of transformations, given specifically as

$$
\triangle_{a_{1} \ldots a_{n}}^{(n)}=A_{u}^{-1} B^{a_{1}} A_{u}^{-1} B^{a_{2}} \ldots A_{u}^{-1} B^{a_{n}}(0,1), \text { where } B=\left(\begin{array}{ll}
1 & 1 \\
0 & 1
\end{array}\right) .
$$

By an easy induction argument, each such interval is contained in the unit interval, and for positive integers $n$ we have

$$
\triangle_{a_{1} \ldots a_{n+1}}^{(n+1)} \subset \triangle_{a_{1} \ldots a_{n}}^{(n)}
$$


Observe that for $a \in V_{u}$ the Möbius transformation $A_{u}^{-1} B^{a}$ maps $(0,1)$ homeomorphically onto $\triangle_{a}^{(1)}$. Thus for any $x \in \triangle_{a}^{(1)}$ there is a unique $y \in(0,1)$ with $x=A_{u}^{-1} B^{a}(y)$. Then

$$
T_{u}(x)=\left\langle A_{u} A_{u}^{-1} B^{a}(y)\right\rangle=\langle y+a\rangle=y .
$$

It follows that $T_{u}$ restricted to $\triangle_{a}^{(1)}$ is the bijection $B^{-a} A_{u}$ mapping the interval onto $(0,1)$, which therefore naturally extends to a homeomorphism of $\bar{\Delta}_{a}^{(1)}$ onto $I$.

Suppressing reference to the particular $u$ or $a_{i}$ we shall write

$$
C_{n}=B^{-a_{n}} A_{u} \ldots B^{-a_{1}} A_{u} .
$$

The notation $T^{n}$ will always refer to the $n$th iterate of the map $T$. The obvious extension of the above by induction gives

Proposition 1. For each $n \geq 1$ and $a_{1}, \ldots, a_{n} \in V_{u}$, the restriction of $T_{u}^{n}$ to $\triangle_{a_{1} \ldots a_{n}}^{(n)}$ is the Möbius transformation $C_{n}$ mapping $\triangle_{a_{1} \ldots a_{n}}^{(n)}$ onto $(0,1)$ that extends to a homeomorphism of $\bar{\Delta}_{a_{1} \ldots a_{n}}^{(n)}$ to $[0,1]$. Similarly, $T: \triangle_{a_{1} \ldots a_{n}}^{(n)} \rightarrow \triangle_{a_{2} \ldots a_{n}}^{(n-1)}$ is the Möbius transformation $B^{-a_{1}} A_{u}$, which extends to a homeomorphism of the closures.

It is clear from the above that the intervals $\triangle_{a_{1} \ldots a_{n}}^{(n)}$ are the maximal open intervals on which the transformation $T_{u}^{n}$ is a homeomorphism. They are sometimes referred to in the literature as intervals of monotonicity. Given a finite sequence of integers $a_{1}, a_{2}, \ldots, a_{n} \in V_{u}$, define the finite $u$-continued fraction expansion

$$
\left[a_{1}, a_{2}, \ldots, a_{n}\right]_{u}=A_{u}^{-1} B^{a_{1}} A_{u}^{-1} B^{a_{2}} \ldots A_{u}^{-1} B^{a_{n}} A_{u}^{-1}(\infty) \in[0,1] .
$$

It follows immediately from the proposition that $T_{u}$ acts as a shift on finite $u$ continued fraction expansions by $T\left[a_{1}, a_{2}, \ldots, a_{n}\right]_{u}=\left[a_{2}, \ldots, a_{n}\right]_{u}$. If we write

$$
C_{n}^{-1} A_{u}^{-1}=\left(\begin{array}{cc}
p_{n} & r_{n} \\
q_{n} & s_{n}
\end{array}\right)
$$

then the expansion may be associated with a "fraction" as follows: $\left[a_{1}, a_{2}, \ldots, a_{n}\right]_{u}$ $=C_{n}^{-1} A_{u}^{-1}(\infty)=\frac{p_{n}}{q_{n}}$. Again, although the explicit reference has been supressed, it is important to keep in mind that the matrix (9) does depend on the digits $a_{1}, a_{2}, \ldots, a_{n}$ and the parameter $u$. For technical reasons we define $p_{0}=0$ and $q_{0}=1$. Also be aware that with this approach $p_{n}$ and $q_{n}$ are not always nonnegative.

Since $C_{n}$ maps $\bar{\Delta}_{a_{1} \ldots a_{n}}^{(n)}$ homeomorphically onto $I, C_{n}^{-1}(0)$ and $C_{n}^{-1}(1)$ are the endpoints of the interval $\triangle_{a_{1} \ldots a_{n}}^{(n)}$. If $u$ is Gauss-like, then $A_{u}^{-1}(\infty)=0$ and if $u$ is Renyi-like, then $A_{u}^{-1}(\infty)=1$. Therefore we have the following values for the endpoints:

$$
\begin{aligned}
& C_{n}^{-1}(0)= \begin{cases}{\left[a_{1}, a_{2}, \ldots, a_{n}\right]_{u}=\frac{p_{n}}{q_{n}}} & \text { if } u \text { is Gauss-like, } \\
{\left[a_{1}, a_{2}, \ldots, a_{n}-1\right]_{u}} & \text { if } u \text { is Renyi-like. }\end{cases} \\
& C_{n}^{-1}(1)= \begin{cases}{\left[a_{1}, a_{2}, \ldots, a_{n}+1\right]_{u}} & \text { if } u \text { is Gauss-like, } \\
{\left[a_{1}, a_{2}, \ldots, a_{n}\right]_{u}=\frac{p_{n}}{q_{n}}} & \text { if } u \text { is Renyi-like. }\end{cases}
\end{aligned}
$$

Note that $\left[a_{1}, a_{2}, \ldots, a_{n} \pm 1\right]_{u}$ is defined as in (18) above, even though it is possible that $a_{n} \pm 1 \notin V_{u}$. 
2.3. The partition by intervals of montonicity and infinite continued fractions. For $n \geq 1$ define $\mathbb{Q}_{u}^{(n)}=\left\{x \in I \mid T_{u}^{m}(x)=0\right.$ for some $\left.m \leq n\right\}$, which we shall call the preimages of zero of order $n$. Let $\mathbb{Q}_{u}=\bigcup_{n=1}^{\infty} \mathbb{Q}_{u}^{(n)}$ be the full set of preimages of 0 . Given $n \geq 1$, define the partition $P_{n}$ of $(0,1)$ consisting of the non-empty cylinder sets of level $n$. Actually, this is only a partition modulo a countable set of points, and more precisely we have

Proposition 2. For each integer $n \geq 1$

$$
[0,1]=\left(\bigcup_{a_{1}, a_{2}, \ldots, a_{n} \in V_{u}} \Delta_{a_{1}, a_{2}, \ldots, a_{n}}^{(n)}\right) \cup \mathbb{Q}_{u}^{(n)} .
$$

Proof. We argue by induction. First suppose $n=1$. The transformation $A_{u}$ maps $[0,1]$ to either $[0, \infty) \cup\{\infty\}$ or $(-\infty, 0] \cup\{\infty\}$ in $\mathbb{R} \cup\{\infty\}$. $A_{u}[0,1]$ therefore consists of $\infty$, some integers and intervals of the form $(k, k+1)$ for some $k \in \mathbb{Z}$. Take the fractional part to get $T_{u}$, thereby sending the integers and $\infty$ to 0 , while each interval $(k, k+1)$ is mapped to $(0,1)$. In other words, each of the open intervals in the complement of $\mathbb{Z}$ in $A_{u}[0,1]$ is the image under $A_{u}$ of a cylinder $\Delta_{k}^{(n)}$ and all such cylinders must be accounted for. The result thus holds for $n=1$.

Suppose the proposition holds for some positive integer $n$. By the induction hypothesis we can write

$$
[0,1]=\left(\bigcup_{a_{2}, \ldots, a_{n+1} \in V_{u}} \Delta_{a_{2}, \ldots, a_{n+1}}^{(n)}\right) \cup \mathbb{Q}_{u}^{(n)},
$$

where we have taken the liberty of indexing the $u$-digits from 2 to $n+1$. Recall that by Proposition 1 the restriction of $T_{u}$ to $\Delta_{a_{1}}^{(1)}$ has a homeomorphic inverse $T_{u}^{-1}=A_{u}^{-1} B^{a_{1}}$ mapping $(0,1)$ onto $\Delta_{a_{1}}^{(1)}$. Then, beginning with what we already know for $n=1$,

$$
\begin{gathered}
{[0,1]=\left(\bigcup_{a_{1} \in V_{u}} \Delta_{a_{1}}^{(1)}\right) \cup \mathbb{Q}_{u}^{(1)}=\left(\bigcup_{a_{1} \in V_{u}} A_{u}^{-1} B^{a_{1}}(0,1)\right) \cup \mathbb{Q}_{u}^{(1)}} \\
=\left(\bigcup_{a_{1} \in V_{u}} A_{u}^{-1} B^{a_{1}}\left(\left(\bigcup_{a_{2}, \ldots, a_{n+1} \in V_{u}} \Delta_{a_{2}, \ldots, a_{n+1}}^{(n)}\right) \cup \mathbb{Q}_{u}^{(n)} \backslash\{0,1\}\right)\right) \cup \mathbb{Q}_{u}^{(1)} \\
=\left(\bigcup_{a_{1}, a_{2}, \ldots, a_{n+1} \in V_{u}} \Delta_{a_{1}, a_{2}, \ldots, a_{n+1}}^{(n)}\right) \cup T_{u}^{-1}\left(\mathbb{Q}_{u}^{(n)} \backslash\{0,1\}\right) \cup \mathbb{Q}_{u}^{(1)},
\end{gathered}
$$

proving the proposition.

By analogy to the classical case, $u=(1,0)$, we shall refer to $\mathbb{Q}_{u}$ as the $u$-rational numbers and to its complement in $I$ as the $u$-irrational numbers. In the classical case this notation agrees with the commonly used dichotomy of the reals. This is no longer true for the backward continued fractions.

It was shown in [11] that all of the maps $T_{u}$ we consider have well-defined derivatives on $(0,1)$ and are eventually expanding. In particular, the second derivative is always bounded below by a constant larger than one. A simple consequence of this is that there is a value $\lambda_{u}>1$ and a constant $K_{u} \geq 0$ so that for any $n>1$ the 
length of an interval $\triangle^{(n)}$ at level $n$ satisfies

$$
\left|\triangle^{(n)}\right|<K_{u} \lambda_{u}^{-n}
$$

Therefore given an infinite sequence of $u$-digits $\left\{a_{n}\right\}_{n=1}^{\infty}$, there is a sequence of nested closed intervals $\left\{\bar{\triangle}_{a_{1} \ldots a_{n}}^{(n)}\right\}_{n=1}^{\infty}$ with lengths converging to zero. Thus, since one endpoint of $\bar{\triangle}_{a_{1} \ldots a_{n}}^{(n)}$ is $\frac{p_{n}}{q_{n}}$, it makes sense to define the value of the infinite $u$-continued fraction

$$
\left[a_{1}, a_{2}, \ldots\right]_{u}=\lim _{n \rightarrow \infty}\left[a_{1}, a_{2}, \ldots, a_{n}\right]_{u}=\lim _{n \rightarrow \infty} \frac{p_{n}}{q_{n}} \in[0,1] .
$$

If $x$ has an infinite $u$-expansion, then

$$
x=\left[a_{1}, a_{2}, \ldots\right]_{u}=\bigcap_{k=1}^{\infty} \bar{\Delta}_{a_{1} \ldots a_{k}}
$$

and consequently

$$
T_{u}(x)=\bigcap_{k=1}^{\infty} T_{u} \bar{\Delta}_{a_{1} \ldots a_{k}}=\bigcap_{k=2}^{\infty} \bar{\Delta}_{a_{2} \ldots a_{k}}=\left[a_{2}, a_{3}, \ldots\right]_{u} .
$$

This shows that $T_{u}$ will always act as a shift on $u$-continued fraction expansions.

Given a $u$-irrational number $x \in(0,1)$, there is an infinite sequence of $u$-digits $\left\{a_{i}\right\}_{i=1}^{\infty}$ so that $x$ lies in each of the intervals $\triangle_{a_{1} \ldots a_{n}}^{(n)}$. Since one of the endpoints of $\triangle_{a_{1} \ldots a_{n}}^{(n)}$ is $\frac{p_{n}}{q_{n}}, \quad x=\lim _{n \rightarrow \infty} \frac{p_{n}}{q_{n}}$.

From the above it also follows that a $u$-irrational number lies in the interior of a unique interval at each level and consequently the $u$-expansion is unique.

What has been proved above is now collected together as a proposition.

Proposition 3. Each u-irrational $x$ has a unique, infinite u-expansion. The continued fraction expansion of the u-irrational $x$ has the form $\left[a_{1}, \ldots, a_{n}, \ldots\right]$ if and only if $x \in \triangle_{a_{1} \ldots a_{n}}^{(n)}$ for each integer $n>0$, and similarly $x=\left[a_{1}, a_{2}, \ldots\right]_{u}$ if and only if $T_{u}^{n-1}(x) \in \triangle_{a_{n}}$ for each integer $n>0$. Furthermore, $T_{u}$ acts as a shift map on u-continued fraction expansions.

The fractions $\frac{p_{n}}{q_{n}}$ are called the $u$-convergents of $x$.

There are basic properties of the $u$-continued fractions and their approximations that remain to be elucidated. At this point it is surprisingly easy to derive some metrical results about the $u$-continued fractions which parallel the classical case. With the techniques developed later, in Section 3, it will become possible to prove more of the basic non-metrical properties of the approximations.

\section{INVARIANT MEASURES AND BASIC METRICAL RESULTS}

In an earlier paper by one of the authors the transformations $T_{u}(x)$ were studied from a purely dynamic point of view [11]. In particular, it was shown that the transformations $T_{u}(x)$ have invariant probability measures $\mu_{u}$, equivalent to Lebesgue measure and defined by the densities

$$
\mu_{u}(x)= \begin{cases}\frac{c_{u}}{x+k} & \text { if } u \text { is Gauss-like, } \\ \frac{c_{u}}{x+k-1} & \text { if } u \text { is Renyi-like, }\end{cases}
$$

where $c_{u}=\operatorname{sgn}(k)\left(\log \left|\frac{k+1-m}{k-m}\right|\right)^{-1}$. Furthermore, $T_{u}$ is ergodic with respect to $\mu_{u}$. 
The following proposition is proved in much the same way as its classical analogues are in [3] and [9]. The case where $m=1$ and $k$ is a positive integer was proved in [10].

Proposition 4. For all $u \in \Psi$ and for almost all $u$-irrational $x=\left[a_{1}, a_{2}, \ldots\right]_{u} \in$ $[0,1]$,

$$
\lim _{n \rightarrow \infty} \frac{1}{n}\left(a_{1}+\ldots+a_{n}\right)= \pm \infty
$$

where the sign agrees with the sign of $k$ and

$$
\lim _{n \rightarrow \infty} \sqrt[n]{\alpha_{1} \ldots \alpha_{n}}=\prod_{i=2}^{\infty}\left(\frac{(i+|k|)^{2}}{(i+|k|)^{2}-1}\right)^{\frac{\operatorname{sgn}(k) \log i}{\log \left|\frac{k+1-m}{k-m}\right|}}
$$

where $\alpha_{i}=a_{i}+1$ if $a_{i} \geq 0$ and $\alpha_{i}=\left|a_{i}\right|$ if $a_{i}<0$.

Proof. We suppose $u$ is Gauss-like with $k>0$. The other cases are similar. Define the functions $\alpha_{n}:[0,1) \rightarrow \mathbb{N}$, where $\alpha_{n}(x)=\alpha_{n}$ for $u$-irrational $x=\left[a_{1}, a_{2}, \ldots\right]_{u}$ and $\alpha_{n}$ is as defined above. Also, define the truncation of $\alpha_{1}$ for $L>1$ by $b_{L}(x)=$ $\alpha_{1}(x)$ if $x \in \bigcup_{|i| \leq L} \Delta_{i}^{(1)}$ and $b_{L}(x)=L$ otherwise. Then for almost all $x$

$$
\liminf _{n \rightarrow \infty} \frac{1}{n} \sum_{i=1}^{n} \alpha_{i}=\liminf _{n \rightarrow \infty} \frac{1}{n} \sum_{i=0}^{n-1} \alpha_{1}\left(T^{i} x\right) \geq \lim _{n \rightarrow \infty} \frac{1}{n} \sum_{i=0}^{n-1} b_{L}\left(T^{i} x\right)
$$

Applying the Birkoff Ergodic Theorem [3, 9] to the function $b_{L}$, we see that the right-hand sum above is equal to

$$
\int_{0}^{1} b_{L}(x) \mu_{u}(x) d x \geq C_{u} \int_{0}^{1} b_{L}(x) d x \geq C_{u} \sum_{i=0}^{L}(i+1)\left|\Delta_{i}^{(1)}\right|
$$

for some constant $C_{u}>0$. Then by definition (6) the length of cylinder at level one is $\left|\Delta_{i}^{(1)}\right|=\left|A_{u}^{-1} B^{i}(0)-A_{u}^{-1} B^{i}(1)\right|=\left|\frac{k}{(i+k)(i+1+k)}\right|$ and consequently for all $L>0$, the right-hand side of equation (14) is bounded below by $C_{u} \sum_{i=0}^{L}\left|\frac{(i+1) k}{(i+k)(i+1+k)}\right|$. This sum diverges as $L$ goes to infinity. That proves the first assertion of the proposition.

In order to prove the second assertion of the proposition, take the logarithm of the left-hand side and apply the Ergodic Theorem. This yields

$$
\lim _{n \rightarrow \infty} \frac{1}{n} \sum_{k=1}^{n} \log \alpha_{n}=\lim _{n \rightarrow \infty} \frac{1}{n} \sum_{k=0}^{n-1} \log \alpha_{1}\left(T^{k} x\right)=\int_{0}^{1} \log \alpha_{1}(x) \mu_{u}(x) d x
$$

Arguing as above, it is clear that the integral can be bounded above by a convergent sum of the form $K_{u} \sum_{i=0}^{\infty} \log (i+1)\left|\Delta_{i}^{(1)}\right|=K_{u} \sum_{i=1}^{\infty}\left|\frac{k \log (i+1)}{(i+k)(i+1+k)}\right|$ for some $K_{u}>0$, 
which justifies the use of the Ergodic Theorem. Compute

$$
\begin{gathered}
\int_{0}^{1} \log \alpha_{1}(x) \mu_{u}(x) d x=c_{u} \sum_{i=0}^{\infty} \log (i+1) \int_{\Delta_{i}^{(1)}} \frac{d x}{x+k} \\
=c_{u} \sum_{i=0}^{\infty} \log (i+1) \int_{A_{u}^{-1} B^{i}(1)}^{A_{u}^{-1} B^{i}(0)} \frac{d x}{x+k}=c_{u} \sum_{i=0}^{\infty} \log (i+1) \int_{\frac{k}{i+1+k}}^{\frac{k}{i+k}} \frac{d x}{x+k} \\
=c_{u} \sum_{i=0}^{\infty} \log (i+1)\left[\log \left(\frac{k}{i+k}+k\right)-\log \left(\frac{k}{i+1+k}+k\right)\right] \\
=c_{u} \sum_{i=1}^{\infty} \log i\left(\log \frac{(i+k)^{2}}{(i+k-1)(i+k+1)}\right) .
\end{gathered}
$$

Turning the sum into the product of the exponents and simplifying gives

$$
\prod_{i=2}^{\infty}\left(\frac{(i+k)^{2}}{(i+k)^{2}-1}\right)^{c_{u} \log i} .
$$

It is surprising that in the other cases the computations simplify in a similar way.

More could be done with these methods, but the natural automorphic extension, developed in the next section, is a far more powerful tool.

\section{The natural eXtension and its Relation \\ TO DIOPHANTINE APPROXIMATION}

4.1. Basic definitions and properties. For each $u=(k, m) \in \Psi$ define the intervals

$$
J_{u}= \begin{cases}(-\infty,-k] & \text { if } m=0, k>0, \\ {[-k, \infty)} & \text { if } m=0, k<-1, \\ (-\infty, 1-k] & \text { if } m=1, k>1, \\ {[1-k, \infty)} & \text { if } m=1, k<0 .\end{cases}
$$

For a Borel subets $D$ of $I \times J_{u}$ let

$$
\rho(D)=\iint_{D} \frac{d x d y}{(x-y)^{2}}
$$

and define the probability measure $\rho_{u}=c_{u} \rho$, where $c_{u}$ is defined as in Section 3 and satisfies $1 / c_{u}=\rho\left(I \times J_{u}\right)$. Observe that we also have the formula

$$
c_{u}= \begin{cases}\left(\log \left(\frac{|k|+1}{|k|}\right)\right)^{-1} & \text { if } \quad(-1)^{m} k>0, \\ \left(\log \left(\frac{|k|}{|k|-1}\right)\right)^{-1} & \text { if } \quad(-1)^{m} k<0 .\end{cases}
$$

The function $\rho_{u}(x, y)=\frac{c_{u}}{(x-y)^{2}}$ is referred to as the density for the measure $\rho_{u}$. Since $\rho_{u}$ and Lebesgue measure on $\mathbb{R}^{2}$ have the same sets of measure zero, "almost everywhere" statements regarding $\rho_{u}$ or one of its projections to $I$ or $J_{u}$ are true with respect to Lebesgue measure on these sets.

We use $S$ to denote the $\sigma$-algebra of Borel sets for any subset of $\mathbb{R}^{n}$. The results from [11] regarding the natural extension of $T_{u}$ will be stated as a theorem for later use. 
Theorem 1. a) There are sets $I_{u} \subset I$ and $J_{u}^{*} \subset J_{u}$, which differ from $I$ and $J_{u}$ on a countable set, so that the map $\tilde{T}_{u}: I_{u} \times J_{u}^{*} \rightarrow I_{u} \times J_{u}^{*}$ defined by $\tilde{T}_{u}(x, y)=$ $\left(T_{u}(x), A_{u}(y)-\left[A_{u}(x)\right]\right)$ is an automorphism with invariant measure $\rho_{u}$.

b) $\tilde{T}_{u}$ is a Bernoulli automorphism of the probability space $\left(I_{u} \times J_{u}^{*}, \rho_{u}, S\right)$.

The following remarks highlight some elementary but important properties of the map $\tilde{T}_{u}$. In the abstract setting, it is known that the endomorphism $T_{u}$ possesses a natural automorphic extension 9 . The map $\tilde{T}_{u}$ is a particular, concrete realization of that natural automorphic extension, but by a common abuse of notation we shall refer to it as the natural automorphic extension of $T_{u}$.

Remark 1 . As it is defined above $\tilde{T}_{u}$ maps $(0,1) \times\left(J_{u} \cup\{\infty\}\right)$ to $[0,1) \times\left(J_{u} \cup\{\infty\}\right)$ and agrees with the above Bernoulli automorphism off a set of measure zero. In most cases we shall work with this larger domain.

Remark 2. It follows from the definition of $\tilde{T}_{u}$ and Proposition 1 that for $x \in$ $\triangle_{a_{1} \ldots a_{n}}^{(n)}$ and $y \in J_{u} \cup\{\infty\}$

$$
\tilde{T}_{u}^{n}(x, y)=\left(C_{n}(x), C_{n}(y)\right) .
$$

In particular, by restricting the first coordinate of the domain in this way $\tilde{T}_{u}$ becomes a fixed Möbius transformation acting on each of the coordinates. Furthermore, if $x=\left[a_{1}, a_{2}, \ldots\right]_{u}$ is a fixed $u$-irrational, then for each integer $n>0$ the $u$ digits $a_{1}, \ldots, a_{n}$ determine a matrix $C_{n}$, as in equation (7), and for any $y \in J_{u} \cup\{\infty\}$ equation (18) holds.

Remark 3. From a measure theoretic point of view the dynamical systems $\left(\tilde{T}_{u}, I_{u} \times\right.$ $\left.J_{u}^{*}, \rho_{u}\right)$ are not all distinct. Set $\alpha(x, y)=(1-x, 1-y)$. Then conjugacy by the order two measure preserving transformation $\alpha$ induces an isomorphism between the dynamical system with $u=(k, 1)$ and the one with $u=(-k, 0)$. First, note that the set of points $(x, y)$ with $x \notin \mathbb{Q}_{u}$ has full measure and for such $x, A_{u}(x) \notin \mathbb{Z}$. Then on this set of full measure compute

$$
\begin{aligned}
\alpha \circ & \tilde{T}_{(k, 1)} \circ \alpha(x, y)=\left(-\frac{k}{x}+k+1+\left[\frac{k}{x}-k\right],-\frac{k}{y}+k+1+\left[\frac{k}{x}-k\right]\right) \\
& =\left(-\frac{k}{x}+k+1-\left[-\frac{k}{x}+k+1\right],-\frac{k}{y}+k+1-\left[-\frac{k}{x}+k+1\right]\right) \\
& =\left(-\frac{k}{x}+k-\left[-\frac{k}{x}+k\right],-\frac{k}{y}+k-\left[-\frac{k}{x}+k\right]\right)=\tilde{T}_{(-k, 0)}(x, y),
\end{aligned}
$$

where we have used the fact that, for any $a \notin \mathbb{Z}, \quad[a]=-[1-a]$.

Remark 4. It should be mentioned that the explicit realization of the natural extension given by Nakada [15] is another avenue for extending the family $T_{u}$ and might provide an alternative to the approach taken here. It has proved very useful in a variety of other settings; for example see [6, 5, 12. Schweiger has also incorporated Nakada's approach into his theory of fibered systems 20, 21] to achieve metrical results for a variety of continued fraction theories in one dimension and higher.

4.2. Diophantine approximation. Fix $u \in \Psi$. Given $x \in(0,1)$ set $\left(x_{0}, y_{0}\right)=$ $\tilde{T}_{u}(x, \infty)$ and define $\tilde{T}_{u}^{n}\left(x_{0}, y_{0}\right)=\left(x_{n}, y_{n}\right)$. If $x$ is a $u$-irrational, $\left(x_{n}, y_{n}\right)$ is defined for all integers $n \geq 0$. One important measure of the degree to which a $u$-convergent $\frac{p_{n}}{q_{n}}$ approximates $x$ is given in classical form by the value

$$
\theta_{n}=\left|q_{n}\right|\left|q_{n} x-p_{n}\right| \text {. }
$$




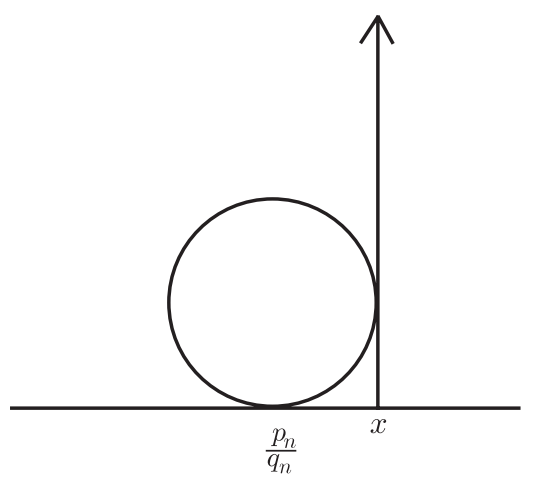

FiguRE 1. The Ford circle of radius $\frac{1}{2 \tau_{n} q_{n}^{2}}$ at $\frac{p_{n}}{q_{n}}$ tangent to $\overline{x \infty}$.

In terms of the natural extension we have

Theorem 2. $\theta_{n}=\frac{1}{\left|x_{n}-y_{n}\right|}$.

Proof. Let $C$ be a Möbius transformation given by the real $2 \times 2$ matrix of determinant $\delta= \pm 1$ as in formula (11). Möbius transformations map circles on the Riemann sphere to circles and therefore the image of the line $y=\tau$ for $\tau>0$ is a circle $S$ through the point $C(\infty)=a / c[2$.

Since the extended real line remains invariant under $C$ and meets $y=\tau$ only at $\infty, S$ is tangent to the real axis at $a / c$. This circle will lie in the upper or lower halfplane depending on whether $\delta=1$ or $\delta=-1$, respectively. Computing the image of a particularly nice point on the line $y=\tau$ under $C$ gives $C\left(-\frac{d}{c}+i \tau\right)=\frac{a}{c} \pm \frac{i}{\tau c^{2}}$, where the sign depends on whether $\delta= \pm 1$. We conclude that $S$ has radius $\frac{1}{2 \tau c^{2}}$.

Let $C$ be the matrix $C_{n+1}$ determined by the $u$-irrational $x=\left[a_{1}, a_{2}, \ldots\right]_{u}$ as in Propositiion 11 and suppose that $C_{n+1}$ has determinant $\delta=1$. Then $C_{n+1}$ takes the vertical ray $\overline{x \infty}$ in the upper half-plane beginning at $x$ to the semi-circle $C_{n+1}(\overline{x \infty})=\widehat{x_{n} y_{n}}$ orthogonal to $\mathbb{R}$ at the points $x_{n}=C_{n+1}(x)$ and $y_{n}=C_{n+1}(\infty)$. The semi-circle $\widehat{x_{n} y_{n}}$ is tangent to the line $y=\tau_{n}$ where $\tau_{n}=\frac{\left|x_{n}-y_{n}\right|}{2}$.

By definition

$$
C_{n+1}^{-1}(\infty)=C_{n}^{-1} A_{u}^{-1} B^{a_{n+1}}(\infty)=C_{n}^{-1} A_{u}^{-1}(\infty)=\frac{p_{n}}{q_{n}},
$$

the $n^{\text {th }} u$-convergent to $x$. By the general considerations in the first paragraph above, $C_{n+1}^{-1}$ takes the line $y=\tau_{n}$ to the circle in the upper half-plane of radius $\frac{1}{2 \tau_{n} q_{n}^{2}}$ which is tangent to $\mathbb{R}$ at the point $p_{n} / q_{n}$. Tangencies are preserved by Mobius transformations, so the ray $\overline{x \infty}$ is tangent to this image circle. See Figure 1. It follows that

$$
\left|x-\frac{p_{n}}{q_{n}}\right|=\frac{1}{2 \tau_{n} q_{n}^{2}} \text { and } \theta_{n}=\frac{1}{2 \tau_{n}}=\frac{1}{\left|x_{n}-y_{n}\right|} .
$$

The case of $\delta=-1$ is similar except that the Möbius transformations $C_{n}$ alternately preserve and interchange the upper and lower half-planes. Although some of the geometric configurations will then be in the lower half-plane, that will have no effect on the result. 
Now, by computing the supremum of $\frac{1}{|x-y|}$ for $(x, y) \in I \times J_{u}$ and noting that $x_{n} \neq 0,1$, we get the following version of the classical result of Dirichlet for the rough approximation of a $u$-irrational number by $u$-rational numbers.

Corollary 1. For each u-irrational number $x \in(0,1)$ the convergents $\frac{p_{n}}{q_{n}}$ (infinite in number) satisfy

$$
\left|x-\frac{p_{n}}{q_{n}}\right|< \begin{cases}\frac{1}{|k| q_{n}^{2}} & \text { if }(-1)^{m} k>0 \\ \frac{1}{(|k|-1) q_{n}^{2}} & \text { if }(-1)^{m} k<0\end{cases}
$$

\section{The DYNAMiCS of $\tilde{T}_{u}$ AND APPLICATIONS}

TO DIOPHANTINE APPROXIMATION

5.1. Distribution under the automorphic extension. One consequence of Theorem 1 is that $\tilde{T}_{u}$ is ergodic. In particular, as a consequence of Birkoff's Ergodic Theorem [9], for almost all $(x, y) \in I \times J_{u}$ and any Borel set $D$ or $f \in L^{1}\left(\rho_{u}\right)$,

$$
\begin{aligned}
& \lim _{n \rightarrow \infty} \frac{1}{n} \#\left\{j \leq n \mid \tilde{T}_{u}^{n}(x, y) \in D\right\}=\rho_{u}(D) \text { and } \\
& \lim _{n \rightarrow \infty} \frac{1}{n} \sum_{i=1}^{n} f\left(\tilde{T}_{u}^{n}(x, y)\right)=\int f d \rho_{u} .
\end{aligned}
$$

What is more surprising is that with some additional hypotheses on $D$ and $f$ the identities (21) hold for almost all $x \in I$ for any $y \in J_{u}$. The remainder of this section will be devoted to the proof of this fact, which is Theorem 3

Lemma 1. Given a u-irrational $x \in(0,1)$ and $\epsilon>0$ there is a value $N$ so that for $n>N$ and $y, y^{\prime} \in J_{u} \cup\{\infty\},\left|\tilde{T}_{u}^{n}(x, y)-\tilde{T}_{u}^{n}\left(x, y^{\prime}\right)\right|<\epsilon$.

Proof. Define the Möbius transformations

$$
M_{(k, 0)}=\left(\begin{array}{cc}
0 & \frac{-k}{\sqrt{|k|}} \\
\frac{1}{\sqrt{|k|}} & 0
\end{array}\right) \text { and } M_{(k, 1)}=\left(\begin{array}{cc}
\frac{1}{\sqrt{|k|}} & \frac{k-1}{\sqrt{|k|}} \\
\frac{1}{\sqrt{|k|}} & \frac{-1}{\sqrt{|k|}}
\end{array}\right) .
$$

$M_{u}$ is an order two homeomorphism which interchanges the intervals $I$ and $J_{u} \cup$ $\{\infty\}$. One easily sees by computation that for $u \in \Psi$ and $n \in V_{u}, \quad M_{u} B^{-n} A_{u} M_{u}^{-1}$ $=(-1)^{m} \operatorname{sgn}(k)\left(B^{-n} A_{u}\right)^{-1}$. Note that multiplication of a matrix by a scalar, as we have above, is term by term and has no effect on the resulting Möbius transformation.

Fix a $u$-irrational $x$ with $u$-expansion $\left[a_{1}, \ldots\right]_{u}$. Then the transformations $C_{n}$ are determined and we can define the associated transformation

$$
\hat{C}_{n}=B^{-a_{1}} A_{u} \ldots B^{-a_{n}} A_{u} .
$$

By Proposition $11 \hat{C}_{n}$ maps $\bar{\Delta}_{a_{n} \ldots a_{1}}^{(n)}$ onto $I$. Then it follows from the previous observation that $M_{u} C_{n} M_{u}^{-1}=\left((-1)^{m} \operatorname{sgn}(k)\right)^{n} \hat{C}_{n}^{-1}$. Applying Remark 2,

$$
\tilde{T}_{u}^{n}\left(\{x\} \times\left(J_{u} \cup\{\infty\}\right)\right)=\left\{C_{n}(x)\right\} \times C_{n}\left(J_{u} \cup\{\infty\}\right) .
$$

The second coordinate can be rewritten as

$$
C_{n}\left(J_{u} \cup\{\infty\}\right)=M_{u} \hat{C}_{n}^{-1} M_{u}\left(J_{u} \cup\{\infty\}\right)=M_{u} \hat{C}_{n}^{-1}(I)=M_{u}\left(\bar{\Delta}_{a_{n} \ldots a_{1}}^{(n)}\right) .
$$


On $(0,1)$ we have $\left|\left(M_{u} A_{u}^{-1} B^{a}\right)^{\prime}\right|=1$ for any $a \in V_{u}$. By equation (10), $N$ can be chosen so that for $n>N,\left|\bar{\Delta}_{a_{n-1} \ldots a_{1}}^{(n-1)}\right|<\epsilon$. Then the interval $C_{n}\left(J_{u} \cup\{\infty\}\right)=$ $M_{u}\left(\bar{\Delta}_{a_{n} \ldots a_{1}}^{(n)}\right)=M_{u} A_{u}^{-1} B^{a_{n}}\left(\bar{\Delta}_{a_{n-1} \ldots a_{1}}^{(n-1)}\right)$ has length less than $\epsilon$. For $y, y^{\prime} \in J_{u} \cup\{\infty\}$ the second coordinates of the points $\tilde{T}_{u}^{n}(x, y)$ and $\tilde{T}_{u}^{n}\left(x, y^{\prime}\right)$ are $C_{n}(y)$ and $C_{n}\left(y^{\prime}\right)$, both of which belong to the interval $M_{u}\left(\bar{\Delta}_{a_{n} \ldots a_{1}}^{(n)}\right)$. The lemma follows.

Theorem 3. For $u \in \Psi$ and for almost all $x \in[0,1]$ the sequence of points $\tilde{T}_{u}^{n}(x, y)$ for positive integers $n$ is distributed in the interior of the region $I \times J_{u}$ according to the density function $\rho_{u}(w, z)$ for all $y \in J_{u} \cup\{\infty\}$. In other words, for any Borel set $\mathbf{B} \subset I \times J_{u}$ which has boundary of measure zero and for almost all $x$,

$$
\lim _{n \rightarrow \infty} \frac{1}{n} \#\left\{j \leq n \mid \tilde{T}_{u}^{n}(x, y) \in \mathbf{B}\right\}=\rho_{u}(\mathbf{B})
$$

for all $y \in J_{u} \cup\{\infty\}$. Furthermore, for any uniformly continuous function $f \in$ $L^{1}\left(\rho_{u}\right)$ and for almost all $x \in I$,

$$
\lim _{n \rightarrow \infty} \frac{1}{n} \sum_{i=1}^{n} f\left(\tilde{T}_{u}^{n}(x, y)\right)=\int f d \rho_{u}
$$

for all $y \in J_{u} \cup\{\infty\}$.

The proof is modeled on arguments in [5] and [12].

Proof. Let $\mathbf{B}$ be a Borel subset of $I \times J_{u}$ whose boundary has measure zero. Consider the $\epsilon$-collar $\mathbf{C}_{\epsilon}$ of $\mathbf{B}$, consisting of all points in the plane within $\epsilon$ of the boundary of $\mathbf{B}$. Define $\mathbf{B}_{\epsilon}^{+}=\mathbf{B} \cup \mathbf{C}_{\epsilon}$ and $\mathbf{B}_{\epsilon}^{-}=\mathbf{B}-\mathbf{C}_{\epsilon}$. Then we have $\mathbf{B}_{\epsilon}^{-} \subset \mathbf{B} \subset \mathbf{B}_{\epsilon}^{+}$.

As a consequence of Lemma 1 , given $x \in(0,1) u$-irrational and $\epsilon>0$, there exists $N^{-}>0$ so that for $n>N^{-}$, if $\tilde{T}_{u}^{n}(x, y) \in \mathbf{B}_{\epsilon}^{-}$for some $y \in J_{u} \cup\{\infty\}$, then $\tilde{T}_{u}^{n}(x, y) \in \mathbf{B}$ for all $y$. Similarly, $N^{+}$can be chosen so that for $n>N^{+}$, if $\tilde{T}_{u}^{n}(x, y) \in \mathbf{B}$ for some $y \in J_{u} \cup\{\infty\}$, then $\tilde{T}_{u}^{n}(x, y) \in \mathbf{B}_{\epsilon}^{+}$for all $y$.

Fix $y^{*} \in J_{u} \cup\{\infty\}$. By the above, for any $(x, y) \in I \times J_{u}$ for which the first and last limits exist,

$$
\begin{aligned}
& \lim _{n \rightarrow \infty} \frac{1}{n} \#\left\{j \leq n \mid \tilde{T}_{u}^{j}(x, y) \in \mathbf{B}_{\epsilon}^{-}\right\} \\
\leq & \liminf _{n \rightarrow \infty} \frac{1}{n} \#\left\{j \leq n \mid \tilde{T}_{u}^{j}\left(x, y^{*}\right) \in \mathbf{B}\right\} \\
\leq & \limsup _{n \rightarrow \infty} \frac{1}{n} \#\left\{j \leq n \mid \tilde{T}_{u}^{j}\left(x, y^{*}\right) \in \mathbf{B}\right\} \\
\leq & \lim _{n \rightarrow \infty} \frac{1}{n} \#\left\{j \leq n \mid \tilde{T}_{u}^{j}(x, y) \in \mathbf{B}_{\epsilon}^{+}\right\} .
\end{aligned}
$$

By the ergodicity of the natural extension and Birkoff's Ergodic Theorem the first and last limits exist for almost all $(x, y) \in I \times J_{u}$ and are, respectively, $\rho_{u}\left(\mathbf{B}_{\epsilon}^{-}\right)$ and $\rho_{u}\left(\mathbf{B}_{\epsilon}^{+}\right)$. Since $\mathbf{B}$ has boundary of measure zero, these two values can be made arbitrarily close by choosing $\epsilon$ small enough. Since $y^{*} \in J_{u} \cup\{\infty\}$ is arbitrary it follows that for almost all $x$

$$
\lim _{n \rightarrow \infty} \frac{1}{n} \#\left\{j \leq n \mid \tilde{T}_{u}^{j}\left(x, y^{*}\right) \in \mathbf{B}\right\}=\rho_{u}(\mathbf{B}),
$$

proving that $\tilde{T}_{u}^{n}(x, y)$ is distributed according to the density $\rho_{u}$. 
Turning now to the second assertion of the theorem, suppose that $\lim _{n \rightarrow \infty} \frac{1}{n} \sum_{i=1}^{n} f\left(\tilde{T}_{u}^{n}(x, y)\right)$ converges to a value $L$. Then for $y^{\prime} \neq y$

$$
\begin{gathered}
\left|\frac{1}{n} \sum_{i=1}^{n} f\left(\tilde{T}_{u}^{n}\left(x, y^{\prime}\right)\right)-L\right| \\
<\left|\frac{1}{n} \sum_{i=1}^{n} f\left(\tilde{T}_{u}^{n}\left(x, y^{\prime}\right)\right)-f\left(\tilde{T}_{u}^{n}(x, y)\right)\right|+\left|\frac{1}{n} \sum_{i=1}^{n} f\left(\tilde{T}_{u}^{n}(x, y)\right)-L\right| .
\end{gathered}
$$

The first sum to the right of the inequality can be made arbitrarily small when $n$ is large, as a consequence of Lemma 1 and the uniform continuity of $f$. The second term goes to zero by hypothesis. Thus the limit with $y^{\prime}$ replacing $y$ also converges to $L$. Since $f$ is integrable, the ergodicity of $\tilde{T}_{u}$ and the Ergodic Theorem give that for almost all $x$ the limit $L$ exists and has the value $\int f d \rho_{u}$.

5.2. Application 1: The Khintchine-Lévy Theorem. As a first application of Theorem 3 we prove the following generalization of the classical result of Khintchine and Lévy [18].

Theorem 4. For $u \in \Psi$ and almost all $x \in I$

$$
\lim _{n \rightarrow \infty} \frac{\log \left|q_{n}\right|}{n}= \begin{cases}\log \sqrt{|k|}-\left(\log \frac{|k|+1}{|k|}\right)^{-1} \mathcal{L}_{2}\left(-\frac{1}{|k|}\right) & \text { if } k(-1)^{m}>0, \\ \log \sqrt{|k|}+\left(\log \frac{|k|}{|k|-1}\right)^{-1} \mathcal{L}_{2}\left(\frac{1}{|k|}\right) & \text { if } k(-1)^{m}<0\end{cases}
$$

where $\mathcal{L}_{2}(z)=\int_{z}^{0} \frac{\log (1-t)}{t} d t$ is the Euler dilogarithm.

The following lemma is useful for relating the limit in Theorem 4 to an ergodic sum.

Lemma 2. For $u \in \Psi$ and $x=\left[a_{1}, a_{2}, \ldots\right]_{u} u$-irrational, the Möbius transformations $C_{n}$ are defined and

$$
C_{n}(\infty)= \begin{cases}\frac{-\operatorname{sgn}(k) \sqrt{|k|} q_{n}}{q_{n-1}} & \text { if } u \text { is Gauss-like, } \\ 1-\operatorname{sgn}(k) \frac{\sqrt{|k|} q_{n}}{q_{n-1}} & \text { if } u \text { is Renyi-like. }\end{cases}
$$

Proof. By taking inverses in formula (9) we get

$$
C_{n}(\infty)=A_{u}^{-1}\left(\begin{array}{cc}
p_{n} & r_{n} \\
q_{n} & s_{n}
\end{array}\right)^{-1}(\infty)= \begin{cases}\frac{k q_{n}}{k q_{n}-s_{n}} & \text { if } u \text { is Gauss-like, } \\
\frac{s_{n}}{s_{n}-k q_{n}} & \text { if } u \text { is Renyi-like. }\end{cases}
$$

In order to simplify these expressions consider the identity

$$
\left(\begin{array}{cc}
p_{n} & r_{n} \\
q_{n} & s_{n}
\end{array}\right)=\left(\begin{array}{ll}
p_{n-1} & r_{n-1} \\
q_{n-1} & s_{n-1}
\end{array}\right) B^{a_{n}} A_{u}^{-1} .
$$

When $u$ is Gauss-like, the right-hand side of (26) becomes

$$
\operatorname{sgn}(k)\left(\begin{array}{cc}
\frac{1}{\sqrt{|k|}}\left(a_{n} p_{n-1}+r_{n-1}\right) & \frac{k}{\sqrt{|k|}}\left(p_{n-1}\left(a_{n}+1\right)+r_{n-1}\right) \\
\frac{1}{\sqrt{|k|}}\left(a_{n} q_{n-1}+s_{n-1}\right) & \frac{k}{\sqrt{|k|}}\left(q_{n-1}\left(a_{n}+1\right)+s_{n-1}\right)
\end{array}\right) .
$$


Comparing lower left-hand entries and solving for $s_{n-1}$ gives $s_{n-1}=\operatorname{sgn}(k) \sqrt{|k|} q_{n}-$ $a_{n} q_{n-1}$. If we substitute this value for $s_{n-1}$ into the expression

$$
s_{n}=\operatorname{sgn}(k) \frac{k}{\sqrt{|k|}}\left(q_{n-1}\left(a_{n}+1\right)+s_{n-1}\right)
$$

that results from equating the lower right-hand entries, we get $s_{n}=\sqrt{|k|} q_{n-1}+k q_{n}$. Substituting this value into formula (25) gives the result when $u$ is Gauss-like. The same type of matrix computation when $u$ is Renyi-like gives $s_{n}=-\sqrt{|k|} q_{n-1}+k q_{n}$, which proves the lemma.

While Lemma 2 is needed for the proof of Theorem 4, there are several other interesting consequences that can be drawn from the lemma and its proof. The following two propositions are generalizations of well-known classical results about continued fractions [18].

Proposition 5. For all $u \in \Psi$ and $x=\left[a_{1}, a_{2}, \ldots\right]_{u}$ u-irrational,

a) $\left|p_{n} q_{n-1}-q_{n} p_{n-1}\right|=\frac{1}{\sqrt{|k|}}$,

b) $\triangle_{a_{1} \ldots a_{n}}^{(n)}$ has the second endpoint $\frac{\sqrt{|k|} p_{n} \pm p_{n-1}}{\sqrt{|k|} q_{n} \pm q_{n-1}}$ with the plus sign if $u$ is Gausslike and the minus sign if $u$ is Renyi-like.

Proof. The values for $r_{n}$ can be determined using the same methods of comparing entries, as in the proof of Lemma 2. The result is $r_{n}=k p_{n} \pm \sqrt{|k|} p_{n-1}$ where, as above, the sign is plus if $u$ is Gauss-like and the sign is minus if $u$ is Renyi-like. The first part then follows by observing that the determinant of the matrix (9) is $p_{n} s_{n}-q_{n} r_{n}=\sqrt{|k|}\left(p_{n} q_{n-1}-q_{n} p_{n-1}\right)$, which has absolute value one.

The second endpoint of $\triangle_{a_{1} \ldots a_{n}}^{(n)}$ is $C_{n}^{-1}(1)=C_{n}^{-1} A_{u}^{-1}(0)=\frac{r_{n}}{s_{n}}$ if $u$ is Gausslike and $C_{n}^{-1}(0)=C_{n}^{-1} A_{u}^{-1}(0)=\frac{r_{n}}{s_{n}}$ if $u$ is Renyi-like. Thus the second part is a consequence of the determinations of $r_{n}$ and $s_{n}$.

Proposition 6. If $x=\left[a_{1}, a_{2}, \ldots\right]_{u}$ is a u-irrational number with convergents $\left[a_{1}, a_{2}, \ldots, a_{n}\right]_{u}=\frac{p_{n}}{q_{n}}$, then

$$
\left[a_{n}, \ldots, a_{1}\right]_{u}=\left\{\begin{array}{cl}
\frac{\sqrt{|k|} q_{n-1}}{q_{n}} & \text { if } u \text { is Gauss-like } \\
1-\frac{\sqrt{|k|} q_{n-1}}{q_{n}} & \text { if } u \text { is Renyi-like. }
\end{array}\right.
$$

Proof. By the properties of $\hat{C}_{n}$ and $M_{u}$ from the proof of Lemma 1,

$$
\begin{aligned}
& {\left[a_{n}, \ldots, a_{1}\right]_{u}=\hat{C}_{n}^{-1} A_{u}^{-1}(\infty)=M_{u} C_{n} M_{u}^{-1} A_{u}^{-1}(\infty)=M_{u} C_{n}(\infty) } \\
= & \left\{\begin{array}{cc}
M_{u}\left(\frac{-\operatorname{sgn}(k) \sqrt{|k|} q_{n}}{q_{n-1}}\right)=\frac{\sqrt{|k|} q_{n-1}}{q_{n}} & \text { if } u \text { is Gauss-like, } \\
M_{u}\left(1-\operatorname{sgn}(k) \frac{\sqrt{|k|} q_{n}}{q_{n-1}}\right)=1-\frac{\sqrt{|k|} q_{n-1}}{q_{n}} & \text { if } u \text { is Renyi-like. }
\end{array}\right.
\end{aligned}
$$

Proof of Theorem 4. Let $g: I \times J_{u} \rightarrow \mathbb{R}$ be a $\rho_{u}$-integrable, uniformly continuous function. As at the beginning of Section 4.2 let $\left(x_{n}, y_{n}\right)=\tilde{T}_{u}^{n+1}(x, \infty)$ for integers $n \geq 0$. Without loss of generality suppose $x$ to be $u$-irrational with $u$-expansion 
$\left[a_{1}, a_{2}, \ldots\right]_{u}$, in which case $y_{n}=C_{n+1}(\infty)$. It follows from Theorem 3 that for almost all $x \in(0,1)$,

$$
\lim _{n \rightarrow \infty} \frac{1}{n} \sum_{j=1}^{n} g\left(x_{j}, y_{j}\right)=\int_{I \times J_{u}} g d \rho_{u} .
$$

If $u$ is Gauss-like, then let $g\left(x_{j}, y_{j}\right)=\log \left|y_{j}\right|$. From Lemma 2 we get $g\left(x_{j}, y_{j}\right)=$ $\log \left|\frac{\sqrt{|k|} q_{j+1}}{q_{j}}\right|$. Unpacking the left-hand side of (27) gives

$$
\begin{gathered}
\lim _{n \rightarrow \infty} \frac{1}{n} \sum_{j=1}^{n}\left(\log \sqrt{|k|}+\log \left|q_{j+1}\right|-\log \left|q_{j}\right|\right), \\
\log \sqrt{|k|}+\lim _{n \rightarrow \infty} \frac{\log \left|q_{n}\right|}{n} .
\end{gathered}
$$

If $u$ is Renyi-like, then take $g\left(x_{j}, y_{j}\right)=\log \left|1-y_{j}\right|=\log \left|\frac{\sqrt{|k|} q_{j+1}}{q_{j}}\right|$, and the left-hand side of (27) is again given by (28).

By first integrating with respect to $x$ and then making a simple substition, e.g. $y=-\frac{1}{x}$ if $m=0$ and $k>0$, the integral in (27) takes the form

$$
\int_{I \times J_{u}} g d \rho_{u}=-c_{u} \int_{0}^{\frac{1}{|k|}} \frac{\log x}{1+(-1)^{m} \operatorname{sgn}(k) x} d x .
$$

Suppose $(-1)^{m} k>0$. After integration by parts the right-hand side of (29) becomes

$$
\begin{gathered}
-c_{u} \int_{0}^{\frac{1}{|k|}} \frac{\log x}{1+x} d x=-c_{u}\left(\left.\log x \log (1+x)\right|_{0} ^{\frac{1}{|k|}}-\int_{0}^{\frac{1}{|k|}} \frac{\log (1+x)}{x} d x\right) \\
=-c_{u}\left(-\log |k| \log \left(\frac{|k|+1}{|k|}\right)-\int_{0}^{-\frac{1}{|k|}} \frac{\log (1-u)}{u} d u\right) \\
=-\left(\log \left(\frac{|k|+1}{|k|}\right)\right)^{-1}\left(-\log |k| \log \left(\frac{|k|+1}{|k|}\right)+\mathcal{L}_{2}\left(-\frac{1}{|k|}\right)\right) .
\end{gathered}
$$

In light of (28) the proof is complete.

If $(-1)^{m} k<0$, then we also have $|k|>1$. Integrate by parts to get

$$
\begin{gathered}
-c_{u} \int_{0}^{\frac{1}{|k|}} \frac{\log x}{1-x} d x=-c_{u}\left(-\left.\log x \log (1-x)\right|_{0} ^{\frac{1}{|k|}}+\int_{0}^{\frac{1}{|k|}} \frac{\log (1-x)}{x} d x\right) \\
=-\left(\log \left(\frac{|k|}{|k|-1}\right)\right)^{-1}\left(\log |k| \log \left(\frac{|k|-1}{|k|}\right)-\mathcal{L}_{2}\left(\frac{1}{|k|}\right)\right) .
\end{gathered}
$$

Combined with (28) the final case of Theorem 4 is proved.

Since by Section 2.2 and Proposition[5 we know the endpoints of an interval of monotonicity, it becomes possible to compute its length:

$$
\left|\triangle_{a_{1} \ldots a_{n}}^{(n)}\right|=\left|\frac{p_{n}}{q_{n}}-\frac{\sqrt{|k|} p_{n} \pm p_{n-1}}{\sqrt{|k|} q_{n} \pm q_{n-1}}\right|=\left|\frac{1}{q_{n}\left(\sqrt{|k|} q_{n} \pm q_{n-1}\right)}\right|,
$$

where the sign depends on the type of $u$ as in Proposition [5] This lead to the following corollary to Theorem 4, which has its classical analogue in [3]. 
Corollary 2. For $u \in \Psi$ and almost all $x \in I$,

$$
\lim _{n \rightarrow \infty} \frac{1}{n} \log \left|\triangle_{a_{1} \ldots a_{n}}^{(n)}\right|= \begin{cases}-\log k+2\left(\log \frac{|k|+1}{|k|}\right)^{-1} \mathcal{L}_{2}\left(-\frac{1}{|k|}\right) & \text { if }(-1)^{m} k>0, \\ -\log k-2\left(\log \frac{|k|}{|k|-1}\right)^{-1} \mathcal{L}_{2}\left(\frac{1}{|k|}\right) & \text { if }(-1)^{m} k<0 .\end{cases}
$$

5.3. Application 2: The rate of approximation. The natural extension can be used to prove the analogues of two other well-known results from the classical theory [3] [5].

Theorem 5. For $u \in \Psi$ and almost all $x \in I$,

1. $\lim _{n \rightarrow \infty} \frac{1}{n} \sum_{i=1}^{n} \frac{\left|x-\frac{p_{i}}{q_{i}}\right|}{\left|x-\frac{p_{i-1}}{q_{i-1}}\right|}= \begin{cases}-1-\left(\log \frac{|k|+1}{|k|}\right)^{-1} \mathcal{L}_{2}\left(-\frac{1}{|k|}\right) & \text { if }(-1)^{m} k>0, \\ 1-\left(\log \frac{|k|}{|k|-1}\right)^{-1} \mathcal{L}_{2}\left(\frac{1}{|k|}\right) & \text { if }(-1)^{m} k<0 .\end{cases}$

2. $\lim _{n \rightarrow \infty} \frac{1}{n} \log \left|x-\frac{p_{n}}{q_{n}}\right|= \begin{cases}-\log k+2\left(\log \frac{|k|+1}{|k|}\right)^{-1} \mathcal{L}_{2}\left(-\frac{1}{|k|}\right) & \text { if }(-1)^{m} k>0, \\ -\log k-2\left(\log \frac{|k|}{|k|-1}\right)^{-1} \mathcal{L}_{2}\left(\frac{1}{|k|}\right) & \text { if }(-1)^{m} k<0 .\end{cases}$

Lemma 3. For $u$-irrational $x \in(0,1)$ and for all integers $n \geq 1$,

$$
\left|x-\frac{p_{i}}{q_{i}}\right|\left|x-\frac{p_{i-1}}{q_{i-1}}\right|^{-1}= \begin{cases}\left|\frac{x_{i-1}}{y_{i-1}}\right| & \text { if } u \text { is Gauss-like, } \\ \left|\frac{1-x_{i-1}}{1-y_{i-1}}\right| & \text { if } u \text { is Renyi-like. }\end{cases}
$$

Proof. We make use of the following well-known identity that holds for any Möbius transformation $C$ and for $z, w \in \mathbb{C}$ with $C(z), C(w) \neq \infty$ :

$$
\left(\frac{C(z)-C(w)}{z-w}\right)^{2}=C^{\prime}(z) C^{\prime}(w)
$$

Suppose $u$ is Gauss-like. From the definitions of $\theta_{i}$ and $\left(x_{i}, y_{i}\right)$, Theorem 2 and Remark 2, we get

$$
\frac{\left|x-\frac{p_{i}}{q_{i}}\right|}{\left|x-\frac{p_{i-1}}{q_{i-1}}\right|}=\frac{\theta_{i}}{\theta_{i-1}}\left(\frac{q_{i-1}}{q_{i}}\right)^{2}=\left|\frac{x_{i-1}-y_{i-1}}{x_{i}-y_{i}}\right| \frac{|k|}{\left(C_{i}(\infty)\right)^{2}}=\left|\frac{x_{i-1}-y_{i-1}}{x_{i}-y_{i}}\right| \frac{|k|}{y_{i-1}^{2}} .
$$

Using the relation $x_{i}=B^{-a_{i}} A_{u}\left(x_{i-1}\right)$ and a similar one for $y_{i}$, we can rewrite the final expression above and then apply the identity (30) with $C=B^{-a_{n}} A_{u}$ to get

$$
\begin{aligned}
& \left|\frac{B^{-a_{i}} A_{u}\left(x_{i-1}\right)-B^{-a_{i}} A_{u}\left(y_{i-1}\right)}{x_{i-1}-y_{i-1}}\right|^{-1} \frac{|k|}{y_{i-1}^{2}} \\
= & \left|\left(B^{-a_{i}} A_{u}\right)^{\prime}\left(x_{i-1}\right)\left(B^{-a_{i}} A_{u}\right)^{\prime}\left(y_{i-1}\right)\right|^{-\frac{1}{2}} \frac{|k|}{y_{i-1}^{2}} .
\end{aligned}
$$

Since $\left(B^{-a_{i}} A_{u}\right)^{\prime}(x)=A_{u}^{\prime}(x)=\frac{-k}{x^{2}}$, the above is equal to

$$
\left(\frac{\left|x_{i-1} y_{i-1}\right|}{|k|}\right) \frac{|k|}{y_{i-1}^{2}}=\left|\frac{x_{i-1}}{y_{i-1}}\right| .
$$

If $u$ is Renyi-like, then $C_{i}(\infty)$ is replaced by $1-C_{i}(\infty)=1-y_{i-1}$ and $\left(B^{-a_{i}} A_{u}\right)^{\prime}(x)$ $=A_{u}^{\prime}(x)=\frac{k}{(1-x)^{2}}$, which also gives the desired result. 
Proof of Theorem [5. Define the function

$$
f_{u}(x, y)= \begin{cases}\left|\frac{x}{y}\right| & \text { if } u \text { is Gauss-like } \\ \left|\frac{1-x}{1-y}\right| & \text { if } u \text { is Renyi-like. }\end{cases}
$$

Then by Theorem 3 and the previous Lemma 3 for almost all $x$

$$
\lim _{n \rightarrow \infty} \frac{1}{n} \sum_{i=1}^{n} \frac{\left|x-\frac{p_{i}}{q_{i}}\right|}{\left|x-\frac{p_{i-1}}{q_{i-1}}\right|}=\lim _{n \rightarrow \infty} \frac{1}{n} \sum_{i=1}^{n} f_{u}\left(\tilde{T}_{u}^{n+1}(x, \infty)\right)=\int_{I \times J_{u}} f_{u} d \rho_{u} .
$$

When $m=0$ and $k>0$ the integral becomes

$$
\begin{gathered}
c_{u} \int_{0}^{1} \int_{-\infty}^{-k} \frac{x}{-y(x-y)^{2}} d y d x=c_{u} \int_{0}^{1} \int_{-\infty}^{-k-x}\left(-\frac{1}{x(v+x)}+\frac{1}{x v}-\frac{1}{v^{2}}\right) d v d x \\
=c_{u} \int_{0}^{1}\left(\frac{-\log k+\log (x+k)}{x}-\frac{1}{x+k}\right) d x
\end{gathered}
$$

where we have substituted $v=y-x$. Now, substituting $t=-x / k$, the above becomes

$$
c_{u}\left(\int_{0}^{-\frac{1}{k}} \frac{\log (1-t)}{t} d t-\log \left(\frac{1+k}{k}\right)\right)=c_{u}\left(-\mathcal{L}_{2}\left(-\frac{1}{k}\right)-\log \left(\frac{1+k}{k}\right)\right)
$$

which is what we expect for part 1 . In the other cases much the same approach using simple substitutions and partial fractions gives the result.

Part 2 would be similarly straightforward except that $\log f_{u}$ is far from being absolutely continuous on $I \times J_{u}$. Let us revisit the proof of the second assertion of Theorem 3. Suppose $u$ is Gauss-like, $f$ is the function $\log f_{u}$ and $x=\left[a_{1}, a_{2}, \ldots\right]$ thereby defining the specific transformations $C_{n}$ for positive integers $n$. Then the first term on the second line of the inequality (24) becomes

$$
\begin{gathered}
\left|\frac{1}{n} \sum_{i=1}^{n} f\left(\tilde{T}_{u}^{n}\left(x, y^{\prime}\right)\right)-f\left(\tilde{T}_{u}^{n}(x, y)\right)\right| \\
=\left|\frac{1}{n} \sum_{i=1}^{n} \log \right| \frac{C_{n}(x)}{C_{n}\left(y^{\prime}\right)}|-\log | \frac{C_{n}(x)}{C_{n}(y)}||=\left|\frac{1}{n} \sum_{i=1}^{n} \log \right| \frac{C_{n}(y)}{C_{n}\left(y^{\prime}\right)}|| .
\end{gathered}
$$

It follows from Lemma 1 that $\lim _{n \rightarrow \infty} \log \left|\frac{C_{n}(y)}{C_{n}\left(y^{\prime}\right)}\right|=0$ and consequently that the limit of the above sum is also zero. That proves the second assertion of Theorem 3 with the revised assumption that $f$ is the function $\log f_{u}$, as defined above, with $u$ Gauss-like. There is little modification required to prove it when $u$ is Renyi-like.

To prove part 2 of Theorem 5 we work with the function $\log f_{u}$. By the above strengthening of Theorem 3 and Lemma 3, for almost all $x$,

$$
\lim _{n \rightarrow \infty} \frac{1}{n} \log \left|x-\frac{p_{n}}{q_{n}}\right|=\lim _{n \rightarrow \infty} \frac{1}{n} \sum_{i=1}^{n} \log \left(\frac{\left|x-\frac{p_{i}}{q_{i}}\right|}{\left|x-\frac{p_{i-1}}{q_{i-1}}\right|}\right)=\int_{I \times J_{u}} \log f_{u} d \rho_{u} .
$$


When $m=1$ and $k>1$ the integral becomes

$$
\begin{aligned}
& c_{u} \int_{0}^{1} \int_{-\infty}^{1-k} \log \left(\frac{1-x}{1-y}\right) \frac{1}{(x-y)^{2}} d y d x \\
& =c_{u} \int_{-1}^{0} \int_{-\infty}^{-k} \log \left(\frac{-x}{-y}\right) \frac{1}{(x-y)^{2}} d y d x,
\end{aligned}
$$

where the obvious substitution has been made. Then we seperate the integrand into two pieces and integrate each seperately to get

$$
\begin{aligned}
& c_{u} \int_{-1}^{0}\left(\int_{-\infty}^{-k} \frac{\log (-x)}{(x-y)^{2}} d y-\int_{-\infty}^{-k} \frac{\log (-y)}{(x-y)^{2}} d y\right) d x \\
= & c_{u} \int_{-1}^{0}\left(\frac{\log (-x)}{(x+k)}-\frac{\log k}{(x+k)}+\frac{\log k}{x}-\frac{\log (x+k)}{x}\right) d x .
\end{aligned}
$$

With a little manipulation this becomes

$$
c_{u}\left(\frac{1}{k} \int_{-1}^{0} \frac{\log \left(-\frac{x}{k}\right)}{\left(\frac{x}{k}+1\right)} d x-\frac{1}{k} \int_{-1}^{0} \frac{\log \left(1+\frac{x}{k}\right)}{\left(\frac{x}{k}\right)} d x\right) .
$$

Substituting $v=-\frac{x}{k}$ in both integrals results in

$$
-c_{u}\left(\int_{\frac{1}{k}}^{0} \frac{\log v}{1-v} d v\right)-c_{u}\left(\int_{\frac{1}{k}}^{0} \frac{\log (1-v)}{v} d v\right) .
$$

The first integral was computed in the proof of Theorem 4 and is

$$
c_{u}\left(\log |k| \log \left(\frac{|k|-1}{|k|}\right)-\mathcal{L}_{2}\left(\frac{1}{|k|}\right)\right) ;
$$

the second is exactly $-c_{u} \mathcal{L}_{2}\left(\frac{1}{k}\right)$. A similar approach works in the other cases.

Remark 5. With the function $f_{u}$ as defined in the beginning of the proof above, Theorem 5 actually shows that for almost all $x \in(0,1)$, for any $y \in J_{u} \cup\{\infty\}$ the ergodic sums

$$
\lim _{n \rightarrow \infty} \frac{1}{n} \sum_{i=1}^{n} f_{u}\left(\tilde{T}_{u}^{n+1}(x, y)\right) \text { and } \lim _{n \rightarrow \infty} \frac{1}{n} \sum_{i=1}^{n} \log f_{u}\left(\tilde{T}_{u}^{n+1}(x, y)\right)
$$

converge to the values given respectively in parts 1 and 2 of the theorem.

5.4. Application 3: Entropy ties it all together and $\frac{1}{\mathbf{n}} \log \boldsymbol{\theta}_{\mathbf{n}}$. Let $h(T)$ denote the measure theoretic entropy of the transformation $T[9,14]$. Rohlin's entropy formula [14, 19 ]

$$
h\left(T_{u}\right)=\int_{0}^{1} \log \left|T_{u}^{\prime}(x)\right| d \mu_{u}
$$

can be applied to compute the entropy of the transformations $T_{u}$. Combined with Theorems 4 and 5 we have

Corollary 3. For $u \in \Psi$ and for almost all $x$,

$$
h\left(T_{u}\right)=2 \lim _{n \rightarrow \infty} \frac{\log \left|q_{n}\right|}{n}=-\lim _{n \rightarrow \infty} \frac{1}{n} \log \left|x-\frac{p_{n}}{q_{n}}\right|=\lim _{n \rightarrow \infty} \frac{1}{n} \log \left|\triangle_{a_{1} \ldots a_{n}}^{(n)}\right| .
$$


Proof. Formula (31) is easily manipulated to give

$$
h\left(T_{u}\right)=-2 c_{u} \int_{0}^{\frac{1}{|k|}} \frac{\log x}{1+(-1)^{m} \operatorname{sgn}(k) x} d x-\log |k| .
$$

The first equality in the corollary follows from equations (27) and (29). The second equality follows by equating Theorem 4 with part 2 of Theorem 5 , with the appropriate multiples inserted.

A similar result appears in [10] for the cases $u=(1, n)$, where $n$ is a positive integer.

Observe that from the second equality in Corollary 3 we get

$$
0=\lim _{n \rightarrow \infty}\left(\frac{1}{n} \log \left|x-\frac{p_{n}}{q_{n}}\right|+2 \frac{\log \left|q_{n}\right|}{n}\right)=\lim _{n \rightarrow \infty} \frac{1}{n}\left(\log \left(\frac{\theta_{n}}{q_{n}^{2}}\right)+2 \log \left|q_{n}\right|\right),
$$

which simplifies to prove

Corollary 4. For $u \in \Psi$ and almost all $x \in I$,

$$
\lim _{n \rightarrow \infty} \frac{1}{n} \log \theta_{n}=0
$$

\section{REFERENCES}

[1] R.L. Adler, L. Flatto, Geodesic flows, interval maps, and symbolic dynamics. Bull. Amer. Math. Soc. 25 (1991), 229-334. MR 92b:58172

[2] T. Bedford, H. Keane, C. Series, eds., Ergodic Theory, Symbolic Dynamics and Hyperbolic Spaces, Oxford Univ. Press, 1991. MR 93e:58002

[3] C. Billingsley, Ergodic Theory and Information. J. Wiley \& Sons, New York-London, 1965. MR 33:254

[4] P. Billingsley, Convergence of Probability Measures, John Wiley \& Sons, New York-London, 1968. MR 38:1718

[5] W. Bosma, H. Jager and F. Wiedijk, Some metrical observations on the approximation of continued factions. Indag. Math. 45 (1983), 281-299. MR 85f:11059

[6] R. Burton, C. Kraaikamp and T. Schmidt, Natural extensions for the Rosen Fractions. Trans. Amer. Math. Soc. 352 (1999), 1277-1298. MR 2000j:11123

[7] H. Cohn, Representation of Markoff's binary quadratic forms by geodesics on a perforated torus. Acta Arith. 18 (1971), 125-136. MR 44:5277

[8] C. Series, The modular surface and continued fractions. J. London Math. Soc. 31 (1985), 69 - 80. MR 87c:58094

[9] I. P. Cornfeld, S.V. Fomin, and Ya. G. Sinai, Ergodic Theory. Springer-Verlag, BerlinHeidelberg-New York, 1982. MR 87f:28019

[10] K. Gröchenig and A. Haas, Backward continued fractions, invariant measures, and mappings of the interval. Ergodic Th. and Dyn. Sys. 16 (1996), 1241-1274. MR 97m:58114

[11] A. Haas, Invariant Measures and Natural Extensions. Canad. Math. Bull. 45 (2002), 97-108. MR 2002j:37005

[12] H. Jager, The distribution of certain sequences connected with the continued fraction. Indag. Math. 89 (1) (1986), 61-69. MR 87g:11092

[13] A. Ya. Khintchine, Continued Fractions (trans. from 3rd Russian ed. by P. Wymm). P. Noordhoff, Ltd., Groningen. MR 28:5038

[14] R. Mañé, Ergodic Theory and Differentiable Dynamics. Springer-Verlag, Berlin, 1983. MR 88c:58040

[15] H. Nakada, Metrical theory for a class of continued fraction transformations and their natural extensions. Tokyo J. Math. 4 (1981), 399-426. MR 83k:10095

[16] G. J. Rieger, Mischung und Ergodizität bei Kettenbrüchen nach nächsten Ganzen. J. Reine Angew. Math. 310 (1979), 171-181. MR 81c:10066

[17] A. Rényi, Valòs szàmok elöàllitàsàra szölgàlò algoritmusokròl. M. T.A. Mat. ès Fiz. Oszt. Kz̈l. 7 (1957), 265-293. MR 20:4113 
[18] A. Rockett and P. Szüsz, Continued Fractions. World Sci. Pub., Singapore, 1992. MR 93m:11060

[19] V.A. Rohlin, Exact endomorphisms of a Lebesgue space. A.M.S. Transl. 39 (1964), 1-37. MR 37:4234

[20] F. Schweiger, Ergodic Theory of Fibered Systems and Metric Number Theory. Clarendon Press, 1995. MR 97h:11083

[21] F. Schweiger, Multidimensional Continued Fractions. Oxford Univ. Press, 2000.

[22] S.M. Rudolfer and K.M. Wilkinson, A number-theoretic class of weak Bernoulli transformations. Math. Systems Theory 7 (1973), 14-24. MR 48:2100

Department of Mathematics, The University of Connecticut, Storrs, Connecticut 06269-3009

E-mail address: haas@math.uconn.edu

Department of Mathematics, The University of Connecticut, Storrs, Connecticut 06269-3009

E-mail address: molnar@stolaf.edu 